\title{
$1 \quad$ Statistical inference in population genomics
}

2

3

\section{Parul Johri ${ }^{1}$, Charles F. Aquadro ${ }^{2}$, Mark Beaumont ${ }^{3}$, Brian Charlesworth ${ }^{4}$, Laurent Excoffier ${ }^{5}$,} Adam Eyre-Walker ${ }^{6}$, Peter D. Keightley ${ }^{4}$, Michael Lynch ${ }^{1}$, Gil McVean ${ }^{7}$, Bret A. Payseur ${ }^{8}$, Susanne P. Pfeifer ${ }^{1}$, Wolfgang Stephan ${ }^{9}$, and Jeffrey D. Jensen ${ }^{1}$

${ }^{1}$ School of Life Sciences, Arizona State University, Tempe, US

${ }^{2}$ Department of Molecular Biology and Genetics, Cornell University, Ithaca, US

${ }^{3}$ School of Biological Sciences, University of Bristol, Bristol, UK

${ }^{4}$ Institute of Evolutionary Biology, School of Biological Sciences, University of Edinburgh, Edinburgh, UK

${ }^{5}$ Institute of Ecology and Evolution, University of Berne, Berne, $\mathrm{CH}$

${ }^{6}$ School of Life Sciences, University of Sussex, Brighton, UK

${ }^{7}$ Big Data Institute, Li Ka Shing Centre for Health Information and Discovery, University of Oxford, Oxford, UK

${ }^{8}$ Laboratory of Genetics, University of Wisconsin-Madison, Madison, US

${ }^{9}$ Natural History Museum, Berlin, DE

keywords: population genetics, population genomics, statistical inference, model-fitting, positive selection, purifying selection, background selection, selective sweeps, genome scans, demography 


\section{ABSTRACT}

51 The field of population genomics has grown rapidly with the recent advent of affordable, large-

52 scale sequencing technologies. As opposed to the situation during the majority of the 20th

53 century, in which the development of theoretical and statistical population-genetic insights out-

54 paced the generation of data to which they could be applied, genomic data are now being

55 produced at a far greater rate than they can be meaningfully analyzed and interpreted. With this

56 wealth of data has come a tendency to focus on fitting specific (and often rather idiosyncratic)

57 models to data, at the expense of a careful exploration of the range of possible underlying

58 evolutionary processes. For example, the approach of directly investigating models of adaptive

59 evolution in each newly sequenced population or species often neglects the fact that a thorough

60 characterization of ubiquitous non-adaptive processes is a prerequisite for accurate inference. We

61 here describe the perils of these tendencies, present our views on current best practices in

62 population genomic data analysis, and highlight areas of statistical inference and theory that are

63 in need of further attention. Thereby, we argue for the importance of defining a biologically

64 relevant baseline model tuned to the details of each new analysis, of skepticism and scrutiny in

65 interpreting model-fitting results, and of carefully defining addressable hypotheses and

66 underlying uncertainties. 
A brief introduction to population genomic inference

79 Population genomic inference - the use of molecular variation and divergence data to infer

80 evolutionary processes - has become widely embraced and highly utilized in fields ranging from

81 evolutionary biology, to ecology, to anthropology, and to medicine. The underlying questions

82 may be demographic in nature, be it estimating the timing of the peopling of the world (Nielsen

83 et al. 2017) or of viral transmission in a congenitally infected newborn (Renzette et al. 2014);

84 alternatively, they may concern the selective history of specific populations, be it identifying

85 mutations that confer cryptic coloration in species adapting to major post-glacial climatic and

86 geological changes (Harris et al. 2020) or viral drug-resistance to clinical therapeutics (Irwin et

87 al. 2016).

The foundational work allowing for the dissection of these evolutionary processes from

89 levels and patterns of variation and divergence was conducted by Fisher, Wright, and Haldane

90 nearly a century ago (e.g., Fisher 1930; Wright 1931; Haldane 1932; for a historical overview,

91 see Provine 1971). This work demonstrated the possibility of studying evolution at the genetic

92 level, integrating the revolutionary ideas of Darwin (1859) with the turn-of-the-century

93 appreciation of Mendel's (1866) research. However, as famously described by Lewontin (1974),

94 this initial theoretical progress during the first half of the 20th century was "like a complex and

95 exquisite machine, designed to process a raw material that no one had succeeded in mining".

96 With the first 'mining' of population-level molecular variation in the 1960s (see Lewontin 1991),

97 this machine was put to work. The next major steps forward were provided by Kimura and Ohta,

98 who offered a comprehensive framework for studying DNA and protein sequence variation

99 based on these fundamental theoretical insights - the Neutral Theory of Molecular Evolution

100 (Kimura 1968, 1983; Ohta 1973) - an advance for which molecular biology also provided 
101 support (King \& Jukes 1969). Despite some claims to the contrary (Kern \& Hahn 2018), Kimura

102 and Ohta's initial postulates have since been largely validated (Walsh \& Lynch 2018; Jensen et

103 al. 2019), and have provided a means to interpret observed molecular variation and divergence

104 within the context of constantly occurring evolutionary processes including mutation, genetic

105 drift, and purifying selection. While ascribing an important role for positive selection on the level

106 of phenotypic evolution (consistent with Darwin's initial notions), the Neutral Theory

107 hypothesizes that at the genetic level beneficial mutations are rare compared to the much larger

108 input of neutral, nearly neutral, and deleterious mutations constantly raining down on the

109 genomes of all species. Accordingly, positive selection per nucleotide is rare compared to

110 genetic drift and purifying selection. The significant effects on evolution at linked sites caused

111 by fitness-altering mutations have been described in detail in the decades since Kimura's initial

112 formulation (Maynard Smith \& Haigh 1974; Charlesworth et al. 1993; reviewed in Charlesworth

$113 \&$ Jensen 2021).

114 With this framework and the availability of datasets to which it could be applied,

115 statistical approaches for analyzing molecular data began to proliferate, frequently employing

116 some form of neutral expectation as a null model. A wide range of rather sophisticated statistical

117 machinery is now available for reconstructing histories of population size change, population

118 subdivision and migration (e.g., Ray \& Excoffier 2009; Beichman et al. 2018), for identifying

119 beneficial mutations based on patterns associated with selective sweeps (e.g., Booker et al. 2017;

120 Stephan 2019), for quantifying the distribution of fitness effects (DFE) of newly arising

121 mutations (e.g., Eyre-Walker \& Keightley 2007; Bank et al. 2014a), as well as for estimating

122 rates of mutation (e.g., Keightley \& Halligan 2009; Keightley 2012; Lynch et al. 2016) and

123 recombination (e.g., Stumpf \& McVean 2003; Auton \& McVean 2012; Spence \& Song 2019). 
124 These approaches operate in a variety of statistical frameworks (see Beaumont et al. 2002;

125 Beaumont \& Rannala 2004; Schraiber \& Akey 2015), and utilize various aspects of the data -

126 including the frequencies of variants in a sample (the site frequency spectrum, SFS), associations

127 between variants (linkage disequilibrium, LD), and/or between-species levels and patterns of

128 divergence at contrasted site classes (e.g., synonymous versus non-synonymous sites).

Challenges of model-choice and parameter-fitting

131 The growing variety of statistical approaches and associated software implementations presents a

132 dizzying array of choices for any given analysis; although many approaches share the same aims,

133 there also exist important differences. For example, some approaches require a relatively high-

134 level of coding ability to implement while others may be applied in easy-to-use software

135 packages; while some are well-tested and justified by population-genetic theory, others are not.

136 Moreover, even the process of translating raw sequencing data into the allele calls and genotypes

137 used as input for these approaches is accompanied by uncertainty that depends on sequencing

138 quality and coverage, availability of a reference genome, and choice of variant calling and

139 filtering strategies (Han et al. 2014; Pfeifer 2021). Adding to this complexity, it has become

140 increasingly clear that demographic estimation may be highly biased when selection and

141 recombination-associated biased gene conversion are neglected (Ewing \& Jensen 2016; Pouyet

142 et al. 2018), whereas estimates of selection intensity and recombination rate may be highly

143 biased when neglecting demographic effects (Dapper \& Payseur 2018; Rousselle et al. 2018;

144 Johri et al. 2020). This creates a circular problem when commencing any new analysis: one

145 needs information about the demographic history to estimate parameters of recombination and

146 selection, while at the same time one needs information about recombination and selection to 
147 estimate the demographic history. An additional challenge, and a frustration for many, is that

148 there is no single 'best approach'; the correct analysis tools to use, and indeed which questions

149 can be answered at all, depend entirely on the details of the organism under study (Myers et al.

150 2008). Specifically, biological parameters that vary among species - including evolutionary

151 parameters (e.g., effective population size $\left(N_{e}\right)$, mutation rates, recombination rates, and

152 population structure and history), genome structure (e.g., the distribution of functional sites along

153 the genome), and life history traits (e.g., mating system) - must all be considered in order to

154 define addressable hypotheses and optimal approaches.

155 Beyond these initial considerations, a more difficult issue often emerges. Namely, very

156 different models may be found to provide a good fit to the observed data (e.g., Harris et al. 2018;

157 and see Louca \& Pennell 2020 for a phylogenetic perspective on the topic). In other words,

158 particular parameter combinations may be found under competing models that are all capable of

159 predicting the observed patterns of variation. For example, assuming neutrality, one may match

160 an empirical observation at a locus by fitting the timing, severity, and duration of a population

161 bottleneck; or alternatively, assuming a constant population size, by fitting the rate and mean

162 strength of selective sweeps. This fact alone implies a simple truism: the ability to fit the

163 parameters of one's preferred model to data does not alone represent proof of biological reality.

164 Rather, it suggests that this model is one - out of potentially very many - that represents a viable

165 hypothesis, which should be further examined via subsequent analyses or experimentation.

166 Examples abound of enthusiastic promotion of a single preferred model, only to be

167 tempered by subsequent demonstrations of the fit of alternative and often simpler / more

168 biologically realistic models. For example, the view that segregating alleles may be commonly

169 maintained by balancing selection (Ford 1975) was tempered by the realization that genetic drift 
170 is often a sufficient explanation (Kimura 1983), and the view that genome-wide selective sweeps

171 on standing variation are pervasive (Garud et al. 2015; Schrider \& Kern 2017) was tempered by

172 the realization that neutral population histories can result in similar patterns (Harris et al. 2018).

173 While one may readily find such examples of using episodic or hypothesized processes to fit

174 large-scale data patterns by neglecting to define expectations arising from common and certain-

175 to-be-occurring processes, determining which models to evaluate, and how to interpret the fit of

176 a model and its alternatives, are challenges for all researchers. To better illustrate this point,

177 Figure 1 presents three scenarios (constant population size with background selection, constant

178 population size with background selection and selective sweeps, and a population bottleneck

179 with background selection and selective sweeps), and provides the fit of each of those scenarios

180 to two incorrect models (population size change assuming strict neutrality, and recurrent

181 selective sweeps assuming constant population size). As shown, each scenario can be well-fit by

182 both incorrect models, with selective sweeps and population bottlenecks generally being

183 confounded, as well as background selection and population growth, as has been described

184 several times before (e.g., Barton 2000; Poh et al. 2014; Ewing \& Jensen 2016; Johri et al.

185 2021).

187 Constructing an appropriate baseline model for population genomic analysis

188 The somewhat disheartening exercise depicted in Figure 1 naturally raises the questions of

189 whether, and if so how, accurate evolutionary inferences can be extracted from DNA sequences

190 sampled from a population. The first point of importance in this regard is that the starting point

191 for any genomic analysis should be the construction of a biologically-relevant baseline model,

192 which includes the processes that must be occurring and shaping levels and patterns of variation 
193 and divergence across the genome. This model should include mutation, recombination,

194 reassortment, and gene conversion (as applicable), purifying selection acting on functional

195 regions and its effects on linked variants (i.e., background selection: Charlesworth et al. 1993,

196 1995; Charlesworth 2013), as well as genetic drift as modulated by, amongst other things, the

197 demographic history and geographic structure of the population. Depending on the organism of

198 interest, there may be other significant biological components to include based on mating

199 system, progeny distributions, ploidy, and so on. It is thus helpful to view this baseline model as

200 being built from the ground up for any new data analysis. Importantly, the point is not that these

201 many parameters need to be fully understood in a given population in order to perform any

202 evolutionary inference, but rather that they all require consideration, and that the effects of

203 uncertainties in their underlying values on downstream inference can be quantified.

204 However, even prior to considering any biological processes, it is important to investigate

205 the data themselves. Firstly, there exists an evolutionary variance associated with the myriad of

206 potential realizations of a stochastic process, as well as the statistical variance introduced by

207 finite sampling. Secondly, it is not advisable to compare one's empirical observation which may

208 include missing data, variant calling or genotyping uncertainty (e.g., effects of low coverage),

209 masked regions (e.g., regions in which variants were omitted due to low mappability and/or

210 callability), and so on, against either an analytical or simulated expectation that lacks those

211 considerations and thus assumes optimal data resolution (Pfeifer 2017). The dataset may also

212 involve a certain ascertainment scheme, either for the variants surveyed (Nielsen 2004), or given

213 some pre-defined criteria for investigating specific genomic regions (e.g., regions representing

214 genomic outliers with respect to a chosen summary statistic; Thornton \& Jensen 2007). For the

215 sake of illustration, Figure 2 follows the same format as Figure 1, but considers two scenarios: 
216 population growth with background selection and selective sweeps, and the same scenario

217 together with data ascertainment (in this case, an under-calling of the singleton class). As shown,

218 due to the changing shape of the frequency spectra, neglecting to account for this ascertainment

219 can greatly affect inference, considerably modifying the fit of both the incorrect demographic

220 and incorrect recurrent selective sweep models to the data. Hence, if sequencing coverage is such

221 that rare mutations are being excluded from analysis, due to an inability to accurately

222 differentiate genuine variants from sequencing errors, the model used for subsequent testing

223 should consequently also ignore these variants. Similarly, if multiple regions are masked in the

224 empirical analysis due to problems such as alignment difficulties, the expected patterns of LD

225 that are observable under any given model may be affected. Furthermore, while the added

226 temporal dimension of time-series data has recently been shown to be helpful for various aspects

227 of population genetic inference (Malaspinas et al. 2012; Foll et al. 2015; Ferrer-Admetlla et al.

228 2016; Lynch \& Ho 2020), such data in no way sidestep the need for an appropriate baseline

229 model, but simply requires the development of a baseline that matches the temporal sampling. In

230 sum, as these factors can greatly affect the power of planned analyses and may introduce biases,

231 the precise details of the dataset (e.g., region length, extent and location of masked regions, the

232 number of callable sites, and ascertainment) and study design (e.g., sample size and single time-

233 point versus time-series data) should be directly matched in the baseline model construction.

234 With these concerns having been satisfied, the first biological addition will logically be

235 the mutation rate and mutational spectrum. For a handful of commonly studied species, both the

236 mean of, and genomic heterogeneity in, mutation rates have been quantified via mutation-

237 accumulation lines and/or pedigree studies (Pfeifer 2020a). However, even for these species,

238 ascertainment issues remain complicating (Smith et al. 2018), variation amongst individuals may 
be substantial (Ness et al. 2015), and estimates only represent a temporal snapshot of rates and

240 patterns that are probably changing over evolutionary time-scales and may be affected by the

241 environment (Lynch et al. 2016; Maddamsetti \& Grant 2020). In organisms lacking experimental

242 information, often the best available estimates come either from a distantly related species or

243 from molecular clock-based approaches. Apart from stressing the importance of implementing

244 either of the experimental approaches in order to further refine mutation-rate estimates for such a

245 species of interest, it is noteworthy that this uncertainty can also be modeled. Namely, if proper

246 estimation has been performed in a closely related species, one may quantify the expected effect

247 on observed levels of variation and divergence of higher and lower rates. The variation in

248 possible data observations induced by this uncertainty is thus now part of the underlying model.

249 The same logic follows for the next parameter addition(s): crossing over / gene conversion, as

250 applicable for the species in question. For example, for a subset of species, per-generation

251 crossing rates in $\mathrm{cM}$ per $\mathrm{Mb}$ have been estimated by comparing genetic maps based on crosses or

252 pedigrees with physical maps (e.g., Kong et al. 2002; Cox et al. 2009; Comeron et al. 2012). In

253 addition, recombination rates scaled by the effective population size have also been estimated

254 from patterns of LD (e.g., Auton et al. 2012; Pfeifer 2020b) - though this approach typically

255 requires assumptions about evolutionary processes that may be violated (e.g., Dapper \& Payseur

256 2018). As with mutation, the effects on inference of changing the recombination rate - whether

257 estimated for the species of interest or a closely related species - can be modeled.

258 The next additions to the baseline model construction are generally associated with the

259 greatest uncertainty - the demographic history of the population, and the effects of direct and

260 linked purifying selection effects. This is a difficult task given the virtually infinite number of

261 potential demographic hypotheses (e.g., Chikhi et al. 2010); furthermore the interaction of 
selection with demography is inherently non-trivial and difficult to treat separately (e.g., Peischl et al. 2013, 2015; Johri et al. 2021). This realization continues to motivate attempts to jointly

264 estimate the parameters of population history together with the DFE of neutral, nearly neutral,

265 weakly deleterious and strongly deleterious mutations - a distribution which is often estimated in

266 both continuous and discrete forms. One of the first important advances in this area used

267 putatively-neutral synonymous sites to estimate changes in population size based on patterns in

268 the SFS and conditioned on that demography to fit a DFE to non-synonymous sites, which

269 presumably experience considerable purifying selection (Keightley \& Eyre-Walker 2007; Eyre-

270 Walker \& Keightley 2009; Schneider et al. 2011). This step-wise approach may become

271 problematic, however, for organisms in which synonymous sites are not themselves neutral

272 (Lynch 2007; Singh et al. 2007; Zeng \& Charlesworth 2010; Choi \& Aquadro 2016; Long et al.

273 2018), or when the SFS of synonymous sites is affected by background selection, which is

274 probably the case generally given their close linkage to directly selected non-synonymous sites

275 (Pouyet et al. 2018; and see Comeron 2014, 2017).

In an attempt to address some of these concerns, Johri et al. (2020) recently developed an

277 approximate Bayesian computation (ABC) approach that relaxes the assumption of synonymous

278 site neutrality and accounts for background selection effects by simultaneously estimating

279 parameters of the DFE alongside population history. The posterior distributions of the

280 parameters estimated by this approach in any given data application (i.e., characterizing the

281 uncertainty of inference), represent a logical treatment of population size change and purifying /

282 background selection for the purposes of inclusion within this evolutionarily relevant baseline

283 model. That said, the demographic model in this implementation is highly simplified, and

284 extensions are needed to account for more complex population histories. While such simulation- 
based inference (see Cranmer et al. 2020), including ABC, provides one promising platform for joint estimation of demographic history and selection, progress on this front has been made using

287 alternative frameworks as well (Williamson et al. 2005; Ragsdale et al. 2018), and developing

288 analytical expectations under these complex models should remain as the ultimate, if distant,

289 goal. Alternatively, in functionally-sparse genomes with sufficiently high rates of recombination,

290 such that assumptions of strict neutrality are viable for some genomic regions, multiple well-

291 performing approaches have been developed for estimating the parameters of much more

292 complex demographic models (e.g., Gutenkunst et al. 2009; Excoffier et al. 2013; Kelleher et al.

293 2019; Steinrücken et al. 2019). In organisms for which such approaches are applicable (e.g.,

294 certain large, coding-sparse vertebrate and land-plant genomes), this intergenic demographic

295 estimation assuming strict neutrality may helpfully be compared to estimates derived from data

296 in or near coding regions that account for the effects of direct and linked purifying selection

297 (Pouyet et al. 2018; Torres et al. 2018; Johri et al. 2020). For newly studied species lacking

298 functional annotation and information about coding density, following the joint estimation

299 procedure would remain as the more satisfactory strategy in order to account for possible

300 background selection effects.

302 Quantifying uncertainty in model-choice and parameter estimation, investigating potential model

303 violations, and defining answerable questions

304 One of the useful aspects of these types of analyses is the ability to incorporate uncertainty in 305 underlying parameters under relatively complex models, in order to determine the impact of such

306 uncertainty on downstream inference. The computational burden of incorporating variability in

307 mutation and recombination rate estimates, or drawing from the confidence- or credibility- 
intervals of demographic or DFE parameters, may be met with multiple highly-flexible simulation tools (Thornton 2014; Kelleher et al. 2018; Haller \& Messer 2019). These are also useful programs for investigating potential model violations that may be of consequence. For

311 example, if a given analysis for detecting population structure assumes an absence of gene flow,

312 it is possible to begin with one's constructed baseline model, add migration parameters to the

313 model in order to determine the effects of varying rates and directions of migration on the

314 summary statistics being utilized in the empirical analysis, and thereby quantify how a violation

315 of that assumption may affect the subsequent conclusions. Similarly, if an analysis assumes the

316 Kingman coalescent (e.g., a small progeny distribution such that at most one coalescent event

317 occurs per generation), but the organism in question may violate this assumption (i.e., with the

318 large progeny number distributions associated with many plants, viruses, and marine spawners),

319 these distributions may too be modeled in order to quantify potential downstream mis-inference.

To illustrate this point, Figure 3 considers two scenarios of constant population size and

321 strict neutrality but with differing degrees of progeny skew, to demonstrate that a violation of

322 this sort that is not corrected for may result in severely under-estimated population sizes as well

323 as the false-inference of high rates of strong selective sweeps. In this case, the mis-inference

324 arises from the reduction in contributing ancestors under these models, as well as to the fact that

325 neutral progeny skew and selective sweeps may both generate multiple-merger events (Durrett \&

326 Schweinsberg 2004; Hallatschek 2018; Matuszewski et al. 2018; Sackman et al. 2019).

327 Similarly, one may investigate the assumptions of constant mutation or recombination rates

328 when they are in reality variable. As shown in Figure 4, when these rates are assumed constant as

329 is common practice, but in reality vary across the genomic region under investigation, the fit of

330 the (incorrect) demographic and selection models considered may again be substantially 
331 modified. Notably, this rate heterogeneity may inflate the inferred strength of selective sweeps.

332 While Figures 3 and 4 serve as examples, the same investigations may be made for cases such as

333 a fixed selective effect when there is in reality a distribution, neutral and unlinked variants when

334 there is in reality linkage disequilibrium, and so on. Simply put, even if a particular biological

335 process / parameter is not being directly estimated, its consequences can nonetheless be

336 explored.

As detailed in Box 1, with such a model incorporating both biological and stochastic variance as well as statistical uncertainty in parameter estimates, and with an understanding of the role of likely model violations, one may investigate which additional questions / hypotheses can be addressed with the data at hand. By using a simulation approach starting with the baseline model and adding hypothesized processes on top, it is possible to quantify the extent to which models, and the parameters underlying those models, may be differentiated and which result in

343 overlapping or indistinguishable patterns in the data (e.g., Lapierre et al. 2017). For example, if

344 the goal of a given study is to identify recent beneficial fixations in a genome - be they

345 potentially associated with high-altitude adaptation in humans, crypsis in mice, or drug-

346 resistance in a virus - one may begin with the constructed baseline model and simulate selective

347 sweeps under that model. As described in Box 2, by varying the strengths, rates, ages, dominance

348 and epistasis coefficients of beneficial mutations, the patterns in the SFS, LD, and/or divergence

349 that may differentiate the addition of such selective sweep parameters from the baseline

350 expectations can be quantified. Moreover, any intended empirical analyses can be evaluated

351 using simulated data (i.e., the baseline, compared to the baseline + the hypothesis) to define the

352 power and false-positive rates associated. If the differences in resulting patterns cannot be

353 distinguished from the expected variance under the baseline model (in other words, if the power 
354 and false-positive rate of the analyses are not favorable), the hypothesis is not addressable with

355 the data at hand (e.g., Poh et al. 2014). If the results are favorable, this analysis can further

356 quantify the extent to which the hypothesis may be tested; perhaps only selective sweeps from

357 rare mutations with selective effects greater than $1 \%$ and that have fixed within the last $0.1 N_{e}$

358 generations are detectable (see Kim \& Stephan 2002; Przeworski 2002), and any others could not

359 be statistically distinguished from expected patterns under the baseline model. Hence, such an

360 exercise provides a critically essential key for interpreting the resulting data analysis.

In this regard, it is worth mentioning two common approaches that may be viewed as

363 variation that are uniquely and exclusively associated with one particular process, the presence of

364 which could support that model regardless of the various underlying processes and details

365 composing the baseline. For example, Fay \& Wu's (2000) $H$-statistic, capturing an expected

366 pattern of high-frequency derived alleles generated by a selective sweep with recombination, was

367 initially proposed as a powerful statistic for differentiating selective sweep effects from

368 alternative models. Results from the initial application of the $H$-statistic were interpreted as

369 evidence of widespread positive selection in the genome of Drosophila melanogaster. However,

370 Przeworski (2002) subsequently demonstrated that the statistic was characterized by low power

371 to detect positive selection, and that significant values could readily be generated under multiple

372 neutral demographic models. The composite likelihood framework of Kim \& Stephan (2002)

373 provided a significant improvement by incorporating multiple predictions of a selective sweep

374 model, and was subsequently built upon by Nielsen et al. (2005) in proposing the SweepFinder

375 approach. However, Jensen et al. (2005) similarly described low power and high false-positive

376 rates under certain neutral demographic models. The particular pattern of LD generated by a 
377 beneficial fixation with recombination described by Kim \& Nielsen (2004) and Stephan et al.

378 (2006) (and see McVean 2007), was also found to be produced under a more limited range of

379 severe neutral population bottlenecks (Jensen et al. 2007; Crisci et al. 2013). The point here is

380 that the statistics themselves represent important contributions for studying patterns of variation,

381 but in any given empirical application they are impossible to interpret without the definition of

382 an appropriate baseline model and related power and false-positive rates. Thus, the search for a

383 pattern unique to a single evolutionary process is not a work-around, and historically such

384 patterns rarely turn out to be process-specific after further investigation. Even if a 'bullet-proof'

385 test were to be someday constructed, it would not be possible to establish its utility without

386 appropriate modeling, an examination of model violations, and power / sensitivity-specificity

387 analyses. But in reality, the simple fact is that some test statistics and estimation procedures

388 perform well under certain scenarios, but not under others.

The second common strategy involves summarizing empirical distributions of a given

390 statistic, and assuming that outliers of that distribution represent the action of a process of

391 interest, such as positive selection (e.g., Garud et al. 2021). However, such an approach is

392 problematic. To begin with, any distribution has outliers, and there will always exist a $5 \%$ or $1 \%$

393 tail for a chosen statistic under a given model. Consequently, a fit baseline model remains

394 necessary to determine if the observed empirical outliers are of an unexpected severity in the

395 empirical distribution, and if the baseline model together with the hypothesized process has, for

396 example, a significantly improved likelihood. Moreover, only by considering the hypothesized

397 process within the context of the baseline model, may one determine if affected loci (e.g., subject

398 to recent sweeps) would even be expected to reside in the tails of the chosen statistical

399 distribution, which is far from a given (Teshima et al. 2006; Thornton \& Jensen 2007). Hence, 
the approach for which we advocate remains essential for defining expectations, power, and

401 false-positive rates, and thus to interpret the significance of observed empirical outliers. As the

402 appropriate baseline evolutionary model may differ strongly by organism and population, this

403 performance must be carefully defined and quantified for each empirical analysis in order to

404 accurately interpret results.

Closing thoughts

407 When it comes to evolutionary analyses, wanting to answer a question is not necessarily

408 equivalent to being able to answer it. The ability of population genomics to address a hypothesis

409 of interest with a given dataset is something that must be demonstrated, and this may be achieved

410 by constructing a model composed of common biological and evolutionary processes, including

411 the uncertainty in those underlying parameters, as well as the specific features of the dataset at

412 hand. The variation in possible observational outcomes associated with a chosen baseline model,

413 and the ability to distinguish an hypothesized additional evolutionary process from that

414 'background noise', are both quantifiable. Furthermore, even if the model were to be correct,

415 there exists a limit on the precision of estimation imposed by the evolutionary variance in

416 population statistics that requires description, and which no amount of sampling can remove.

417 Demonstrating that multiple models, and/or considerable parameter space within a model,

418 are compatible with the data need not be viewed as a negative or weak finding. Quite the

419 contrary - the honest presentation of such results motivates future theoretical, experimental, and

420 empirical developments and analyses that can further refine the list of competing hypotheses, and

421 this article contains many citations that have succeeded in this vein. At the same time, this

422 analysis can define which degrees of uncertainty are most damaging (e.g., Figures 3 and 4), also 
423 highlighting the simple fact that organisms in which basic biological processes have been better

424 characterized are amenable to a wider-range of potential evolutionary analyses. The impact of

425 uncertainty in these parameters in non-model organisms may motivate taking a step back to first

426 better characterize the basic biological processes such as mutation rates and spectra via mutation-

427 accumulation lines or pedigree studies, in order to improve resolution on the primary question of

428 interest.

Importantly, the framework we describe will also generally identify many models and

430 parameter realizations that are in fact inconsistent with the observed data. This 'ruling-out'

431 process can often be just as useful as model-fitting, and rejecting possible hypotheses is

432 frequently the more robust exercise of the two. The value of this narrowing down, rather than the

433 enthusiastic promotion of individual scenarios, is worthy of heightened appreciation.

434 Nevertheless, all models should not be viewed equally. Decades of work supporting the central

435 tenets of the Neutral Theory (Jensen et al. 2019), high-quality experimental and computational

436 work quantifying mutation rate and recombination rate (e.g., Lynch et al. 2008; Auton \&

437 McVean 2012; Comeron et al. 2012; Ness et al. 2015; Smith et al. 2018; Pfeifer 2020a),

438 constantly improving experimental and theoretical approaches to quantify the neutral and

439 deleterious DFE from natural population, mutation-accumulation, or directed mutagenesis data

440 (e.g., Keightley \& Eyre-Walker 2007; Bank et al. 2014b; Foll et al. 2014; Böndel et al. 2019;

441 Johri et al. 2020), and often historical knowledge (e.g., anthropological, ecological, clinical) of

442 population size change or structure - combined with the fact that all of these processes may

443 strongly shape observed levels and patterns of variation and divergence - justify their status in

444 comprising the appropriate baseline model for genomic analysis. Given this, and particularly

445 once accounting for the inflation of variance contributed by uncertainty in these parameters, 
446 potential model violations, as well as the quantity and quality of data available in any given

447 analysis, it will often be the case that many hypotheses of interest may not be addressable with

448 the dataset and knowledge at hand. However, recognizing that a question cannot be accurately

449 answered, and defining the conditions under which it could become answerable, must be the

450 preference over making unfounded and thus misleading claims. Consistent with this call for

451 caution however, it should equally be emphasized that the fit of a baseline model to data is

452 certainly not inherent evidence that the model encompasses all relevant processes shaping the

453 population. In reality, it is virtually guaranteed not to be all-encompassing, and building these

454 models involves simplifying more complex processes (for a helpful and more general

455 perspective, see Gelman \& Shalizi 2013). When an additional process on top of this model

456 cannot be satisfactorily detected, that may rather be viewed as a statement about statistical

457 identifiability - the inability to distinguish a hypothesized process from other processes that are

458 known to be acting - and in such scenarios, absence of evidence need not be taken as evidence of

459 absence.

While the many considerations described may appear daunting, it is our hope that this

461 may serve as a useful roadmap for future data analyses in population genomics, one that may

462 inform not only the perspectives of authors, but also that of reviewers and editors as well.

463 Helpfully, these strategies can save considerable time, money, and effort prior to the start of

464 empirical data handling, by determining which questions are accessible to the researcher. If a

465 question is addressable, this preliminary analysis can additionally define what types of data are

466 needed; for example, the number of variants or sample size necessary to obtain sufficient power,

467 or how alternative data collections (e.g., temporal samples) could improve resolution. This

468 further highlights the value of defining specific hypotheses and of studying specific patterns as 
opposed to running a general suite of software on each new dataset in the hopes of identifying something of interest - namely, one cannot define power to address an unformulated question.

471 Such hypothesis-driven population genomics has resulted in a number of success stories over the

472 past decade; systems in which specific hypotheses were formed, data was collected for the

473 purpose, detailed population genomic analyses were designed, and ultimately important insights

474 were gained about the evolutionary history of the population in question (e.g., the study of

475 cryptic coloration has proven particularly fruitful in this regard, Harris et al. 2020). One feature

476 common amongst these studies is interdisciplinarity: the utilization of population genetic theory

477 and inference as described here, combined with classical genetic crosses, large-scale field

478 studies, and genetic manipulation in order to connect genotype to phenotype to fitness and to

479 validate statistical inference. Importantly however, without the population genetic framework to

480 define hypotheses, quantify processes contributing to observed variation and divergence,

481 evaluate and distinguish amongst competing models, and define uncertainty and potential biases,

482 these empirical observations alone remain merely descriptive.

\section{METHODS}

486 Both forward- and coalescent-simulations were performed (see below for details) for (1) the

487 inference of demographic history using ABC assuming complete neutrality, (2) the inference of

488 parameters of positive selection using $\mathrm{ABC}$ assuming constant population size, and (3) to obtain

489 test datasets representing different evolutionary scenarios. In all cases, a chromosomal segment

490 of 99,012 bp was simulated with an intron-exon-intergenic structure resembling the $D$.

491 melanogaster genome. Each gene comprised five exons (of $300 \mathrm{bp}$ each) and four introns (of 100 
492 bp each) separated by intergenic regions of length 1,068 bp. Such a construct resulted in a total

493 of 33 genes across the simulated segment. Population parameters were chosen to resemble those

494 from D. melanogaster populations following Campos et al. (2019), assuming an effective

495 population size $\left(N_{\mathrm{e}}\right)$ of $10^{6}$ individuals with a mean mutation rate $(\mu)$ of $4.5 \times 10^{-9}$ per bp/gen

496 and a mean recombination rate $(r)$ of $1 \times 10^{-8}$ per bp/gen. For computational efficiency, all

497 parameters were re-scaled by a factor of 200.

499 Modeling and inference of demographic history

500 A simple demographic history was modeled in which a single population undergoes an

501 instantaneous change from an ancestral size $\left(N_{\text {anc }}\right)$ to a current size $\left(N_{\text {cur }}\right), 100$ generations ago.

502 Priors for both $N_{\text {anc }}$ and $N_{\text {cur }}$ were sampled from a loguniform distribution between 10 and

50350,000 , while priors for $\tau$ were sampled from a loguniform distribution between 10 and $N_{c u r}$.

504 One hundred replicates were simulated for each parameter combination. Simulations required for

505 ABC were performed in msprime v. 0.7 .3 (Kelleher et al. 2016) assuming complete neutrality.

506 Mutation and recombination rates were assumed to be constant across the genome and across

507 replicates.

509 Modeling and inference of positive selection

510 A recurrent selective sweep scenario was modeled in which only neutral and beneficial

511 mutations were assumed to be present, with simulations performed using SLiM v. 3.1 (Haller \&

512 Messer 2019). Introns and intergenic regions were assumed to be neutral, while exons

513 experienced beneficial mutations with fitness effects sampled from an exponential distribution

514 with mean $s$. The two parameters varied were the mean population-scaled strength of selection, 
$515 \gamma=2 N_{a n c} S$, and the proportion of new beneficial mutations, $f_{p o s}$. Priors for these parameters

516 were sampled from a loguniform distribution such that $\gamma \in[0.1,10000]$ and $f_{\text {pos }} \in$

$517[0.00001,0.01]$. For all parameter combinations, the true rate of beneficial substitutions per site

$518\left(d_{a}\right)$ and the true fraction of substitutions due to beneficial mutations $(\lambda$, which is related to the $\alpha$

519 parameter of Eyre-Walker \& Keightley) was calculated using the total number of fixations (as

520 provided by SLiM), which was found to range from 0-0.85 depending on the underlying

521 parameters. Parameter inference was performed for $\gamma$ and $d_{a}$ and the corresponding $\lambda$ was

522 inferred using $\lambda=\frac{d_{a}}{d_{a}+\left(\left(1-f_{\text {pos }}\right) \times \mu \times \text { num of generations }\right)}$, where it was assumed that $1-f_{\text {pos }} \sim 1$.

523 Populations were assumed to have a constant size and comprised of 5000 diploid individuals

524 with constant mutation and recombination rates as specified above. Selection coefficients were

525 re-scaled by the factor 200 and simulations were run for 100,100 generations (i.e., $20 N_{\mathrm{e}}+100$

526 generations).

529 The sample size was set to 100 haploid genomes (or 50 diploid individuals). Under both

530 demographic and selection models described above, all exonic regions were masked and the

531 mean and variance (across replicates) of the following summary statistics were calculated:

532 number of segregating sites, nucleotide site diversity $(\pi)$, Watterson's theta $\left(\theta_{W}\right), \theta_{H}, H^{\prime}$,

533 Tajima's $D$, number of singletons, haplotype diversity, and statistics summarizing $\operatorname{LD}\left(r^{2}, D, D^{\prime}\right)$.

534 All statistics were calculated in non-overlapping sliding windows of $2 \mathrm{~kb}$ using pylibseq v. 0.2.3

535 (Thornton 2003). ABC was performed using the R package "abc" v. 2.1 (Csilléry et al. 2012)

536 using all summary statistics, with "neural net" to account for non-linearity between statistics and 
parameters. A 100-fold cross-validation was used to identify the optimum tolerance level, which was found to be 0.05 .

\section{Simulations of different evolutionary scenarios as 'true scenarios'}

541 To consider more biologically realistic models and evaluate model violations, a number of

542 evolutionary scenarios were simulated (using SLiM) as follows:

543 a) Background selection: Exons experienced deleterious mutations modeled by a discrete

544 DFE comprised of four non-overlapping uniform distributions, representing the

545 effectively neutral $\left(-1<2 N_{a n c} s \leq 0\right)$, weakly deleterious $\left(-10<2 N_{a n c} s \leq-1\right)$,

546 moderately deleterious $\left(-100<2 N_{a n c} s \leq-10\right)$, and strongly deleterious $\left(2 N_{a n c} s \leq\right.$

$547-100$ ) classes of mutations. All four bins were assumed to contribute equally to new

548 mutations (i.e., $25 \%$ of all new mutations belonged to each class of mutation).

b) Positive selection: Exons experienced beneficial mutations with $\gamma=125$ and $f_{\text {pos }}=$ $2.2 \times 10^{-3}$ (modified from Campos \& Charlesworth 2019), resulting in $\lambda \sim 0.35$.

c) Population size change: A population decline was simulated such that the population declined from 5000 to 100 individuals instantaneously 100 generations ago. A population expansion was similarly simulated with parameters $N_{a n c}=5000$ and $N_{c u r}=10000$. A population bottleneck model was also simulated such that $N_{a n c}=N_{c u r}=5000$ and a bottleneck occurred 2000 generations ago with a severity of $1 \%$ and a duration of 100 generations.

d) SNP ascertainment: Genotype error was modeled as an inability to detect the true number of singletons when using low-coverage population-genomic data to call variants (Han et 
al. 2014). To account for this scenario, a random set of singletons, representing a third of all singletons present in the sample, were removed.

e) Progeny skew: A skew in the offspring distribution was modeled as a $\psi$-coalescent (Eldon \& Wakeley 2006; and see Matuszewski et al. 2018; Sackman et al. 2019), such that $5 \%$ and $10 \%$ of the population was replaced by the offspring of a single individual each generation.

f) Variation in mutation and recombination rates across the genome (e.g., McVean et al. 2004; Chan et al. 2012; Penalba \& Wolf 2020): Every $10 \mathrm{~kb}$ of the $100 \mathrm{~kb}$ genomic region considered was assumed to have a different mutation and recombination rate. For every simulated replicate, these rates were sampled from a Gaussian distribution with the same mean as above, and a standard deviation of $0.5 \times$ mean value. Negative values were truncated to 0 .

\section{Posterior checks}

574 showing a simple evaluation of the fit of the inferred posteriors under the incorrect models to

575 the true scenarios under consideration). Specifically, the mean estimates of the inferred

576 parameters were used to simulate the "best-fitting model" in SLiM v. 3.1 (Haller \& Messer

577 2019). Exons were masked and summary statistics were calculated as above in windows of 2

$578 \quad$ kb using pylibseq v.0.2.3 (Thornton 2003). In order to simulate the inferred models of

579 positive selection, $f_{\text {pos }}$ was calculated from $\lambda$ assuming a Wright-Fisher diploid population

580 of size $N$ and a total mutation rate of $\mu_{\text {tot }}$ (which for our purpose is the same as $\mu$ ). Thus, $581 \quad \mu_{b}=f_{\text {pos }} \times \mu_{\text {tot }}$ and $\mu_{\text {neu }}=\left(1-f_{\text {pos }}\right) \times \mu_{\text {tot }}$ where $\mu_{b}$ and $\mu_{\text {neu }}$ are the beneficial and 
584 follows:

585 given that

$$
\lambda=\frac{\# \text { of beneficial subs }}{\# \text { of beneficial subs }+\# \text { of neutral subs }}
$$

588 where,

$$
\text { \#of beneficial subs }=P_{\text {fix }} \times L \times 2 N \mu_{b}
$$

$590 \quad$ and,

$$
\text { \#of neutral subs }=\mu_{n e u} \times L
$$

592 where $L$ is the length of the region being considered and $P_{f i x}$ is the probability of fixation of 593 beneficial mutations, such that

$$
P_{f i x}=\int_{0}^{\infty} \frac{\left(1-e^{-x}\right)}{\left(1-e^{-2 N x}\right)}\left(\frac{e^{-x / \bar{s}}}{\bar{s}}\right) d x
$$

$$
f=\frac{\lambda}{(1-\lambda) P_{f i x} 2 N+\lambda}
$$

600 Statistics were calculated in non-overlapping windows of $2 \mathrm{~kb}$ and confidence intervals (CIs) 601 were calculated as the 0.025 and 0.975 quantiles of the distribution of the statistics. 
607 We would like to thank Mark Kirkpatrick and Kevin Thornton for helpful comments and critical

608 feedback. This work was funded by National Institutes of Health grants R01GM135899 and

609 R35GM139383 to JDJ.

610

611

612 


\section{CITATIONS}

614

Auton, A., A. Fledel-Alon, S.P. Pfeifer, O. Venn, L. Ségurel, T. Street, E.M. Leffler, R. Bowden, I. Aneas, J. Broxholme, P. Humburg, Z. Iqbal, G. Lunter, J. Maller, R.D. Hernandez, C. Melton, A. Venkat, M.A. Nobrega, R. Bontrop, S. Myers, P. Donnelly, M. Przeworski, and G. McVean, 2012. A fine-scale chimpanzee genetic map from population sequencing. Science 336(6078): 193-198.

Auton, A., and G. McVean, 2012. Estimating recombination rates from genetic variation in humans. Methods Mol. Biol. 856: 217-237.

Bank, C., M. Foll, A. Ferrer-Admetlla, G. Ewing, and J.D. Jensen, 2014a. Thinking too positive? Revisiting current methods in population genetic selection inference. Trends Genet. 30(12): 540546.

Bank, C., R.T. Hietpas, A. Wong, D.N.A. Bolon, and J.D. Jensen, 2014b. A Bayesian MCMC approach to assess the complete distribution of fitness effects of new mutations: uncovering the potential for adaptive walks in challenging environments. Genetics 196(3): 841-852.

Barton, N.H., 2000. Genetic hitchhiking. Phil. Trans. R. Soc. B 355(1403): 1553-1562.

Beaumont, M.A., and B. Rannala, 2004. The Bayesian revolution in genetics. Nat. Rev. Genet. 5(4): 251-261.

Beaumont, M.A., W. Zhang, and D.J. Balding, 2002. Approximate Bayesian computation in population genetics. Genetics 162(4): 2025-2035.

Beichman, A.C., E. Huerta-Sanchez, and K.E. Lohmueller, 2018. Using genomic data to infer historic population dynamics of non-model organisms. Annu. Rev. Ecol. Evol. Syst. 49: 433456.

Böndel, K.B., S.A. Kraemer, T.S. Samuels, D. McClean, J. Lachapelle, R.W. Ness, N. Colegrave, and P.D. Keightley, 2019. Inferring the distribution of fitness effects of spontaneous mutations in Chlamydomonas reinhardtii. PLoS Biol. 17(6): e3000192.

Booker, T.R., B.C. Jackson, and P.D. Keightley, 2017. Detecting positive selection in the genome. BMC Biol. 15(1):98.

Campos, J.L., and B. Charlesworth, 2019. The effects on neutral variability of recurrent selective sweeps and background selection. Genetics 212(1): 287-303.

Chan, A.H., P. Jenkins, and Y.S. Song, 2012. Genome-wide fine-scale recombination rate variation in Drosophila melanogaster. PLoS Genet. 8(12): e1003090.

Charlesworth, B., 2013. Background selection 20 years on. The Wilhelmine E. Key 2012 invitational lecture. J. Hered. 104(2): 161-171. 
656 Charlesworth, D., B. Charlesworth, and M.T. Morgan, 1995. The pattern of neutral molecular

657 variation under the background selection model. Genetics 141(4): 1619-1632.

658 Charlesworth, B., and J.D. Jensen, 2021. The effects of selection at linked sites on patterns of 659 genetic variability. Annu. Rev. Ecol. Evol. Syst., in press.

660 Charlesworth, B., M.T. Morgan, and D. Charlesworth, 1993. The effect of deleterious mutations 661 on neutral molecular variation. Genetics 134(4): 1289-1303.

662 Chikhi, L., V.C. Sousa, P. Luisi, B. Goossens, and M.A. Beaumont, 2010. The confounding 663 effects of population structure, genetic diversity and the sampling scheme on the detection and 664 quantification of population size change. Genetics 186(3): 983-995.

665 Choi, J.Y., and C.F. Aquadro, 2016. Recent and long term selection across synonymous sites in 666 Drosophila ananassae. J. Mol. Evol. 83(1-2): 50-60.

667 Comeron, J.M., 2014. Background selection as baseline for nucleotide variation across the 668 Drosophila genome. PLoS Genet. 10(6): e1004434.

669 Comeron, J.M., 2017. Background selection as a null hypothesis in population genomics:

670 insights and challenges from Drosophila studies. Phil. Trans. R. Soc. B 372(1736): 20160471.

671 Comeron, J.M., R. Ratnappan, and S. Bailin, 2012. The many landscapes of recombination in 672 Drosophila melanogaster. PLoS Genet. 8(10): e1002905.

673 Cox, A., C. Ackert-Bicknell, B. Dumont, Y. Ding, J. Tzenova Bell, G. Brockmann, J. Wergedal, 674 C. Bult, B. Paigen, J. Flint, S-W. Tsaih, G. Churchill, and K. Broman, 2009. A new standard 675 genetic map for the laboratory mouse. Genetics 182(4): 1335-1344.

676 Cranmer, K., J. Brehmer, and G. Louppe, 2020. The frontier of simulation-based inference. Proc. Natl. Acad. Sci. USA 117(48): 30055-30062.

Crisci, J., Y.-P. Poh, S. Mahajan, and J.D. Jensen, 2013. The impact of equilibrium assumptions on tests of selection. Front. Genet. 4: 235.

Csilléry K., O. François, and M.G.B. Blum, 2012. abc: an R package for approximate Bayesian

Dapper, A.L., and B.A. Payseur, 2018. Effects of demographic history on the detection of recombination hotspots from linkage disequilibrium. Mol. Biol. Evol. 35(2): 335-353.

690

691

Darwin, C., 1859. On the origin of species by means of natural selection, or the preservation of favoured races in the struggle for life. John Murray, London, UK.

692

Durrett, R. and J. Schweinsberg, 2004. Approximating selective sweeps. Theor. Popul. Biol. 66(2): 129-138. 
Eldon, B., and J. Wakeley, 2006. Coalescent processes when the distribution of offspring number among individuals is highly skewed. Genetics 172(4): 2621-2633.

698

Ewing, G., and J.D. Jensen, 2016. The consequences of not accounting for background selection in demographic inference. Mol. Ecol. 25(1): 135-141. demographic inference from genomic and SNP data. PLoS Genet. 9(10): e1003905.

Eyre-Walker, A., and P.D. Keightley, 2007. The distribution of fitness effects of new mutations.

Eyre-Walker, A., and P.D. Keightley, 2009. Estimating the rate of adaptive molecular evolution in the presence of slightly deleterious mutations and population size change. Mol. Biol. Evol. 26(9): 2097-2108.

Fay, J., and C.-I Wu, 2000. Hitchhiking under positive Darwinian selection. Genetics 155(3): 1405-1413.

Ferrer-Admetlla, A., C. Leuenberger, J.D. Jensen, and D. Wegmann, 2016. An approximate Markov model for the Wright-Fisher diffusion and its application to time series data. Genetics 203(2): 831-846.

Fisher, R.A., 1930. The genetical theory of natural selection. Clarendon Press, Oxford, UK.

Foll, M., Y.-P. Poh, N. Renzette, A. Ferrer-Admetlla, H. Shim, A.-S. Malaspinas, G. Ewing, C. Bank, P. Liu, D. Wegmann, D.R. Caffrey, K.B. Zeldovich, D.N.A. Bolon, J. Wang, T.F. Kowalik, C.A. Schiffer, R.W. Finberg, and J.D. Jensen, 2014. Influenza virus drug resistance: a time-sampled population genetics perspective. PLoS Genet. 10(2): e1004185.

Foll, M., H. Shim, and J.D. Jensen, 2015. A Wright-Fisher ABC-based approach for inferring per-site effective pop
Res. 15(1): 87-98.

Ford, E.B., 1975. Ecological genetics. Chapman and Hall, London, UK. e1005004. 
Gelman, A., and C.R. Shalizi, 2013. Philosophy and the practice of Bayesian statistics. Br J Math Stat Psychol. 66(1): 8-38.

Gutenkunst, R., R. Hernandez, S. Williamson, and C. Bustamante, 2009. Inferring the joint demographic history of multiple populations from multidimensional SNP data. PLoS Genet. 5(10): e1000695.

Haldane, J.B.S., 1932. The causes of evolution. Longmans, London, UK.

Hallatschek, O., 2018. Selection-like biases emerge in population models with recurrent jackpot events. Genetics 210(3): 1053-1073.

Haller, B.C., and P.W. Messer, 2019. SLiM 3: Forward genetic simulations beyond the WrightFisher model. Mol. Biol. Evol. 36(3): 632-637.

Han E., J.S. Sinsheimer, and J. Novembre, 2014. Characterizing bias in population genetic inferences from low-coverage sequencing data. Mol. Biol. Evol. 31(3): 723-735.

Harris, R.B., K. Irwin, M.R. Jones, S. Laurent, R.D.H. Barrett, M.W. Nachman, J.M. Good, C.R. Linnen, J.D. Jensen, and S.P. Pfeifer, 2020. The population genetics of crypsis in vertebrates: recent insights from mice, hares, and lizards. Heredity 124(1): 1-14.

754 adaptive process. Virus Evol. 2(1): vew014.

Jensen, J.D., Y. Kim, V.B. DuMont, C.F. Aquadro, and C.D. Bustamante, 2005. Distinguishing

Jensen, J.D., B.A. Payseur, W. Stephan, C.F. Aquadro, M. Lynch, D. Charlesworth, and B.

Jensen, J.D., K.R. Thornton, C.D. Bustamante, and C.F. Aquadro, 2007. On the utility of linkage disequilibrium as a statistic for identifying targets of positive selection in non-equilibrium populations. Genetics 176(4): 2371-2379.

Johri, P., B. Charlesworth, and J.D. Jensen, 2020. Towards an evolutionarily appropriate null model: jointly inferring demography and purifying selection. Genetics 215(1): 173-192. 
Johri, P., K. Riall, H. Becher, L. Excoffier, B. Charlesworth, and J.D. Jensen, 2021. The impact of purifying and background selection on the inference of population history: problems and prospects. Mol. Biol. Evol. 38(7): 2986-3003.

Keightley, P.D., 2012. Rates and fitness consequences of new mutations in humans. Genetics

774 190(2): 295-304.

Keightley, P.D., and A. Eyre-Walker, 2007. Joint inference of the distribution of fitness effects of deleterious mutations and population demography based on nucleotide polymorphism frequencies. Genetics 177(4): 2251-2261.

Keightley, P.D., and D.L. Halligan, 2009. Analysis and implications of mutational variation. Genetica 136(2): 359-369.

Kelleher J., A.M. Etheridge, and G. McVean, 2016. Efficient coalescent simulation and genealogical analysis for large sample sizes. PLoS Comput. Biol. 12(5): e1004842.

Kelleher, J., K. Thornton, J. Ashander, and P. Ralph, 2018. Efficient pedigree recording for fast population genetics simulation. PLoS Comput. Biol. 14(11): e1006581.

Kelleher, J., Y. Wong, A.W. Wohns, C. Fadil, P.K. Albers, and G. McVean, 2019. Inferring whole-genome histories in large population datasets. Nat. Genet. 51(9): 1330-1338.

Kern, A.D., and M.W. Hahn, 2018. The neutral theory in light of natural selection. Mol. Biol. Evol. 35(6): 1366-1371.

Kim, Y., and R. Nielsen, 2004. Linkage disequilibrium as a signature of selective sweeps.

792 Genetics 167(3): 1513-1524.

793 Kim, Y., and W. Stephan, 2002. Detecting a local signature of genetic hitchhiking along a recombining chromosome. Genetics 160(2): 765-777.

Kimura, M., 1968. Evolutionary rate at the molecular level. Nature 217(5129): 624-626.

Kimura, M., 1983. The neutral theory of molecular evolution. Cambridge Univ. Press,

King, J.L., and T.H. Jukes, 1969. Non-Darwinian evolution. Science 164(3881): 788-798. 
Lapierre, M., A. Lambert, and G. Achaz, 2017. Accuracy of demographic inference from the site frequency spectrum: the case of the Yoruba population. Genetics 206(1): 439-449.

Lewontin, R.C., 1974. The genetic basis of evolutionary change. Columbia Univ. Press, New York.

807 Lewontin, R.C., 1991. Twenty-five years ago in Genetics: electrophoresis in the development of evolutionary genetics: milestone or millstone? Genetics 128(4): 657-662.

Long, H., W. Sung, S. Kucukyildirim, E. Williams, S. Miller, W. Guo, C. Patterson, C. Gregory, C. Strauss, C. Stone, C. Berne, D. Kysela, W. Shoemaker, M. Muscarella, H. Luo, J. Lennon, Y. Brun, and M. Lynch, 2018. Evolutionary determinants of genome-wide nucleotide composition. Nat. Ecol. Evol. 2(2): 237-240.

813 Louca, S., and M.W. Pennell, 2020. Extant timetrees are consistent with a myriad of 814 diversification histories. Nature 580(7804): 502-505.

815 Lynch, M., 2007. The origins of genome architecture. Sinauer Associates, Sunderland, MA.

816 Lynch, M., M.S. Ackerman, J.F. Gout, H. Long, W. Sung, W.K. Thomas, and P.L. Foster, 2016. 817 Genetic drift, selection and the evolution of the mutation rate. Nat. Rev. Genet. 17(11): 704-714.

Lynch, M., and W.-C. Ho, 2020. The limits to estimating population-genetic parameters with temporal data. Gen. Biol. Evol. 12(4): 443-455.

Lynch, M., W. Sung, K. Morris, N. Coffey, C.R. Landry, E.B. Dopman, W.J. Dickinson, K. spectrum of spontaneous mutations in yeast. Proc. Natl. Acad. Sci. USA 105(27): 9272-9277.

Maddamsetti, R., and N.A. Grant, 2020. Divergent evolution of mutation rates and biases in the long-term evolution experiment with Escherichia coli. Gen. Biol. Evol. 12(9): 1591-1603.

Malaspinas, A-S., O. Malaspinas, S.N. Evans, and M. Slatkin, 2012. Estimating allele age and selection coefficient from time-serial data. Genetics 192(2): 599-607.

Matuszewski, M., M.E. Hildebrandt, G. Achaz, and J.D. Jensen, 2018. Coalescent processes with skewed offspring distributions and non-equilibrium demography. Genetics 208(1): 323-338.

Maynard Smith, J. and J. Haigh, 1974. The hitch-hiking effect of a favourable gene. Genet. Res. 23(1): 23-25.

McVean, G., 2007. The structure of linkage disequilibrium around a selective sweep. Genetics 175(3): 1395-1406.

McVean, G., S. Myers, S. Hunt, P. Deloukas, D. Bentley, and P. Donnelly, 2004. The fine-scale structure of recombination rate variation in the human genome. Science 304(5670): 581-584. 
Mendel, G., 1866. Versuche über Pflanzenhybriden. Ver. Naturforsch. Ver. Brünn 4: 3-47.

Myers, S., C. Fefferman, and N. Patterson, 2008. Can one learn history form the allelic spectrum? Theor. Popul. Biol. 73(3): 342-348.

Ness, R.W., A.D. Morgan, V. Radhakrishnan, N. Colegrave, and P.D. Keightley, 2015. Extensive de novo mutation rate variation between individuals and across the genome of Chlamydomonas reinhardtii. Genome Res. 25(11): 1739-1749.

Nielsen, R., 2004. Population genetic analysis of ascertained SNP data. Hum. Genomics 1(3): 218-224.

Nielsen, R., J.M. Akey, M. Jakobsson, J.K. Pritchard, S. Tishkoff, and E. Willerslev, 2017. Tracing the peopling of the world through genomics. Nature 541(7637): 302-310.

Nielsen, R., S. Williamson, Y. Kim, M.J. Hubisz, A.G. Clark, and C.D. Bustamante, 2005. Genomic scans for selective sweeps using SNP data. Genome Res. 15(11): 1566-1575.

Ohta, T., 1973. Slightly deleterious mutant substitutions in evolution. Nature 246(5428): 96-98.

Peischl, S., I. Dupanloup, M. Kirkpatrick, and L. Excoffier, 2013. On the accumulation of deleterious mutations during range expansions. Mol. Ecol. 22(24): 5972-5982.

Peischl, S., M. Kirkpatrick, and L. Excoffier, 2015. Expansion load and the evolutionary dynamics of a species range. Am. Nat. 185(4): E81-E93.

Penalba, J.V., and J.B. Wolf, 2020. From molecules to populations: appreciating and estimating recombination rate variation. Nat. Rev. Genet. 21(8): 476-492.

Pfeifer, S.P., 2017. From next-generation resequencing reads to a high quality variant data set. Heredity 118(2): 111-124.

Pfeifer, S.P., 2020a. Spontaneous mutation rates. In The Molecular Evolutionary Clock. Theory and Practice. Springer Nature.

Pfeifer, S.P., 2020b. A fine-scale genetic map for vervet monkeys. Mol. Biol. Evol. 37(7): 18551865.

Pfeifer, S.P., 2021. Studying mutation rate evolution in primates - the impacts of computational pipeline and parameter choices. GigaScience, in press.

Poh, Y.-P., V. Domingues, H.E. Hoekstra, and J.D. Jensen, 2014. On the prospect of identifying adaptive loci in recently bottlenecked populations. PLoS One 9(11): e110579. 
Pouyet, F., S. Aeschbacher, A. Thiery, and L. Excoffier, 2018. Background selection and biased gene conversion affect more than $95 \%$ of the human genome and bias demographic inferences. eLife 7: e36317.

Provine, W.B., 1971. The origins of theoretical population genetics. University of Chicago Press.

Przeworski, M., 2002. The signature of positive selection at randomly chosen loci. Genetics 160(3): 1179-1189.

Ragsdale, A., C. Moreau, and S. Gravel, 2018. Genomic inference using diffusion models and the allele frequency spectrum. Curr. Opin. Gen. Deve. 53: 140-147.

Ray, N., and L. Excoffier, 2009. Inferring past demography using spatially explicit population genetic models. Hum. Biol. 81(2-3): 141-157.

Renzette, N., L. Gibson, J.D. Jensen, and T.F. Kowalik, 2014. Human cytomegalovirus intrahost evolution - a new avenue for understanding and controlling herpesvirus infections. Curr. Opin. Virol. 8: 109-115.

Rousselle, M., M. Maeva, B. Nabholz, T. Bataillon, and N. Galtier, 2018. Overestimation of the adaptive substitution rate in fluctuating populations. Biol. Lett. 14(5): 20180055.

Sackman, A., R.B. Harris, and J.D. Jensen, 2019. Inferring demography and selection in organisms characterized by skewed offspring distributions. Genetics 211(3): 1019-1028.

Schneider, A., B. Charlesworth, A. Eyre-Walker, and P.D. Keightley, 2011. A method for inferring the rate of occurrence and fitness effects of advantageous mutations. Genetics 189(4): $1427-1437$.

Schraiber, J.G., and J.M. Akey, 2015. Methods and models for unravelling human evolutionary history. Nat. Rev. Genet. 16(12): 727-740.

Schrider, D.R., and A.D. Kern, 2017. Soft sweeps are the dominant mode of adaptation in the human genome. Mol. Biol. Evol. 34(8): 1863-1877.

Singh, N.D., V.L. Bauer DuMont, M.J. Hubisz, R. Nielsen, and C.F. Aquadro, 2007. Patterns of mutation and selection at synonymous sites in Drosophila. Mol. Biol. Evol. 24(12): 2687-2697.

Smith, T.C.A., P.F. Arndt, and A. Eyre-Walker, 2018. Large scale variation in the rate of germline de novo mutations, base composition, divergence and diversity in humans. PLoS Genet. 14(3): e1007254.

Spence, J.P. and Y.S. Song, 2019. Inference and analysis of population-specific fine-scale recombination maps across 26 diverse human populations. Sci. Adv. 5(10): eaaw9206. 
924 Steinrücken, M., J. Kamm, J. Spence, and Y. Song, 2019. Inference of complex population 925 histories using whole-genome sequences from multiple populations. Proc. Natl. Acad. Sci. USA 926 116(34): 17115-17120.

927 Stephan, W., 2019. Selective sweeps. Genetics 211(1): 5-13.

928 Stephan, W., Y.S. Song and C.H. Langley, 2006. Hitchhiking effect on linkage disequilibrium 929 between linked neutral loci. Genetics 172(4): 2647-2663.

930 Stumpf, M.P., and G.A. McVean, 2003. Estimating recombination rates from population-genetic 931 data. Nat. Rev. Genet. 4(12): 959-968.

932

933

934

935

Teshima, K., G. Coop, and M. Przeworski, 2006. How reliable are empirical genome scans for selective sweeps? Genome Res. 16(6): 702-712.

Thornton K., 2003. Libsequence: a C++ class library for evolutionary genetic analysis. Bioinformatics 19(17): 2325-2327.

Thornton, K.R., 2014. A C++ template library for efficient forward-time population genetic simulation of large populations. Genetics 198(1): 157-166.

Thornton, K.R., and J.D. Jensen, 2007. Controlling the false positive rate in multi-locus genome scans for selection. Genetics 175(2): 737-750.

947 Walsh, B., and M. Lynch, 2018. Evolution and selection of quantitative traits. Oxford University 948 Press, Oxford.

949 Williamson, S.H., R. Hernandez, A. Fledel-Alon, L. Zhu, R. Nielsen, and C.D. Bustamante, 950 2005. Simultaneous inference of selection and population growth from patterns of variation in 951 the human genome. Proc. Natl. Acad. Sci. USA 102(22): 7882-7887.

952 Wright, S., 1931. Evolution in Mendelian populations. Genetics 16(2): 97-159.

953 Zeng, K., and B. Charlesworth, 2010. Studying patterns of recent evolution at synonymous sites 954 and intronic sites in Drosophila melanogaster. J. Mol. Evol. 70(1): 116-128. 
bioRxiv preprint doi: https://doi.org/10.1101/2021.10.27.466171; this version posted November 2, 2021. The copyright holder for this preprint (which was not certified by peer review) is the author/funder, who has granted bioRxiv a license to display the preprint in perpetuity. It is made available under aCC-BY-NC-ND 4.0 International license.

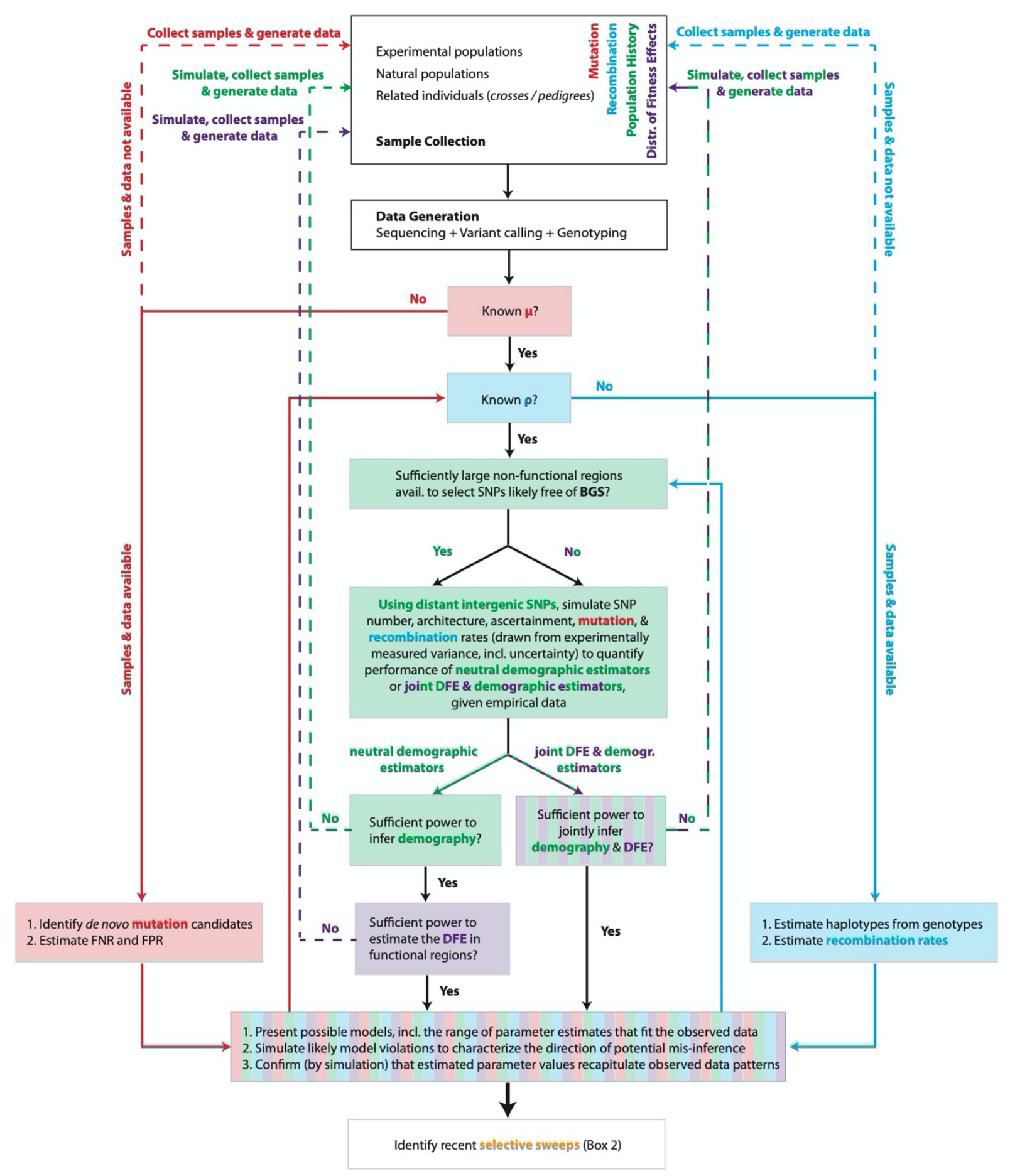

957 Box 1: Diagram of important considerations in constructing a baseline model for genomic analysis. Considerations related to mutation rate are coded in red, recombination rate in blue, demographic history in green, and the distribution of fitness effects in purple - as well as combinations thereof. Beginning from the top with the source of data collected, the arrows suggest a path that is needed to be considered. 


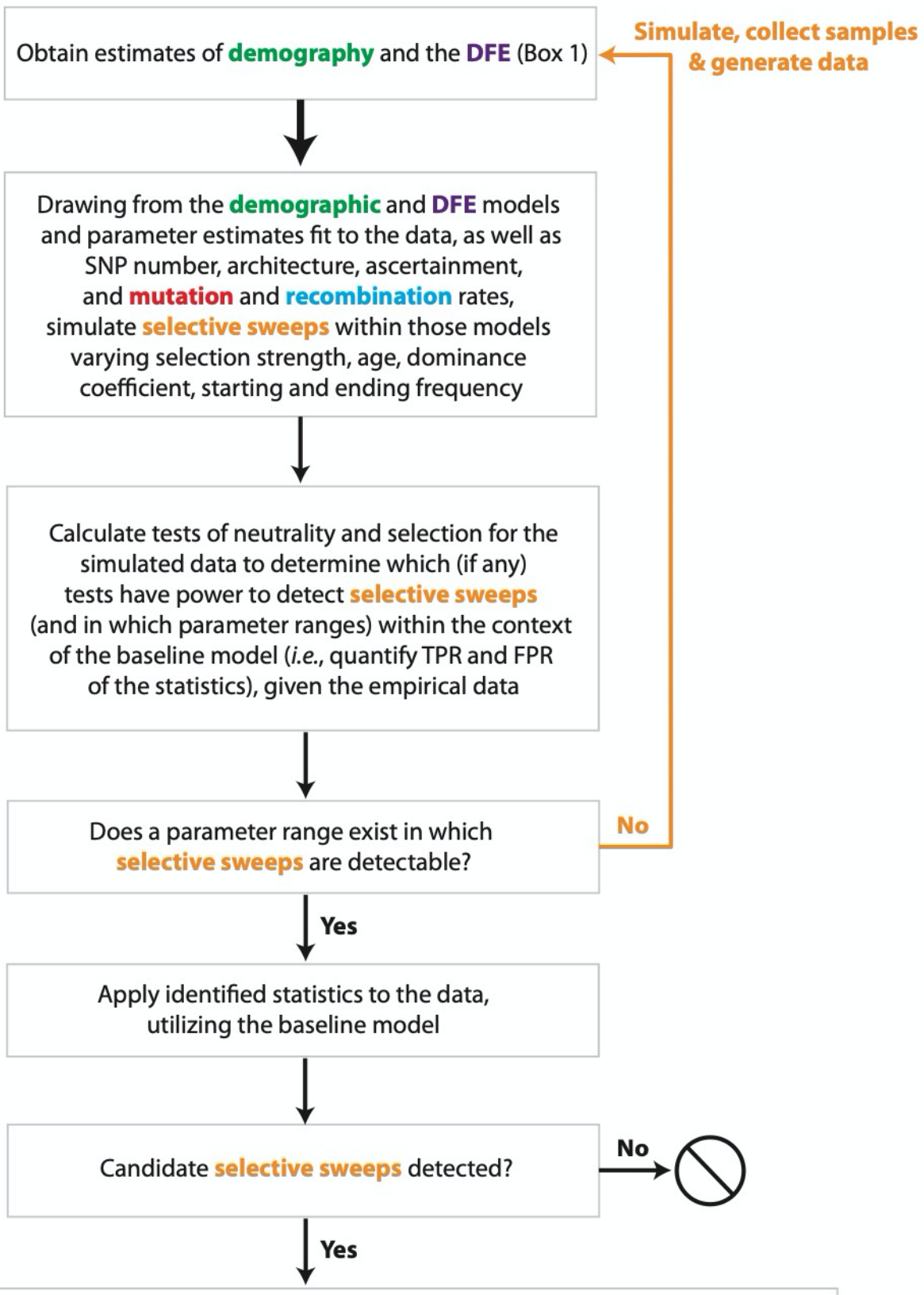

1. Quantify sweep parameters and uncertainty in estimates for candidate loci

2. Simulate likely model violations to characterize the direction of potential mis-inference

3. Confirm (by simulation) that estimated sweep parameters recapitulate observed data patterns

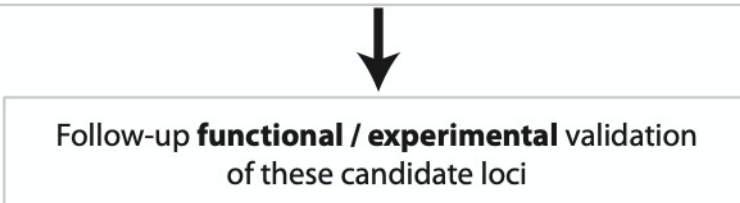

963 Box 2: Diagram of important considerations in detecting selective sweeps. The color scheme 
True
scenario

qm

+ BGS

Eqm

+ BGS

+ Pos

Bottleneck

$+\mathrm{BGS}$

+ Pos

966

967

968

969

970

971

972

973

974

975

976

977

978

979

980

981

982

983

984

985

986

987
Summaries of the data

SFS
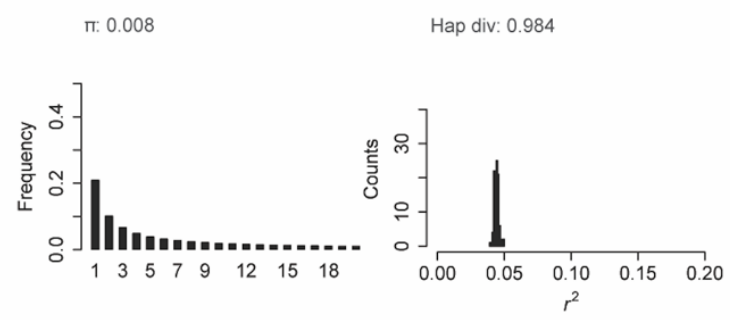

п: 0.004
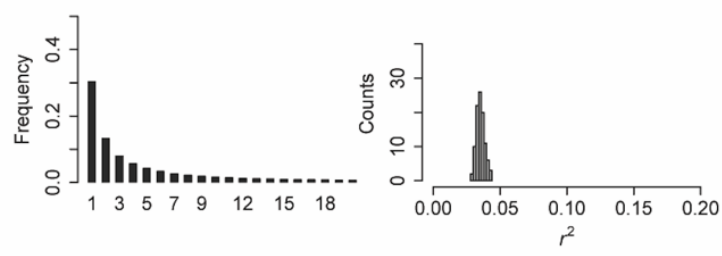

п. 0.002

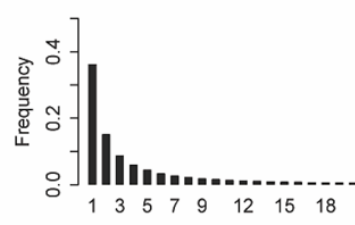

Hap div: 0.916

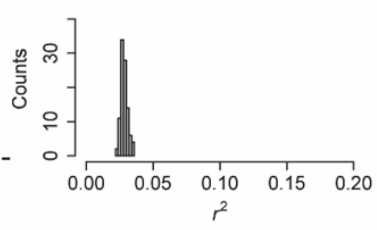

Fit of simulated data to:

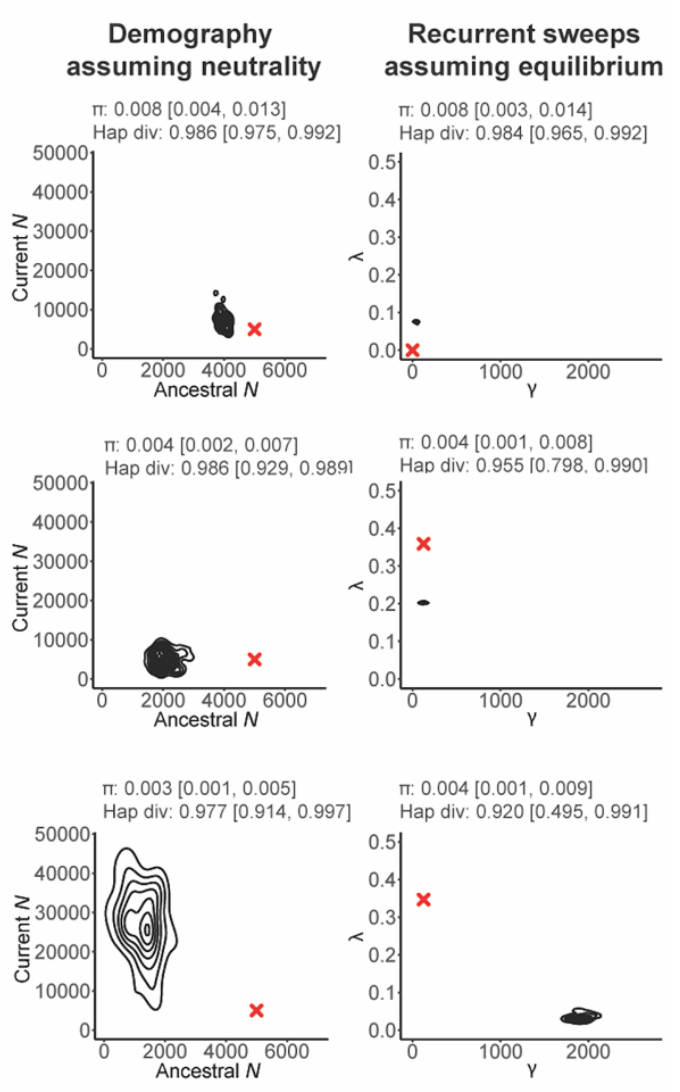

\section{Figure 1. Incorrect models may often readily be fit to a given dataset}

Here we present three scenarios varying from simple to more complex: the first row presents a constant-sized population experiencing background selection (denoted by 'Eqm + BGS'), the second row is the same scenario with the addition of recurrent selective sweeps (denoted by 'Eqm + BGS + Pos'), and the final row adds a population bottleneck (denoted by 'Bottleneck + BGS + Pos'). For each scenario, the resulting site frequency spectra (SFS, truncated to $n=20$ ) and linkage disequilibrium $\left(r^{2}\right)$ distributions are given, together with mean pairwise $(\pi)$ and haplotype diversity. To these simulated data we fit two incorrect models; one assuming all sites are neutral but including a change in population size (with the current size, ancestral size, and time of change being estimated from the data), and a second model in which there are recurrent selective sweeps, no change in population size, and all mutations are assumed to be neutral or beneficial (with a population-scaled beneficial selection coefficient $(\gamma)$ and the fraction of beneficial substitutions $(\lambda)$ being estimated from the data). For each inference panel, the red cross gives the true value, the distribution presents the joint-posterior obtained from the $\mathrm{ABC}$ analysis, and the summary statistics given above the posteriors represent the mean values, and the range from the $95 \%$ CIs, obtained from posterior checks. In all cases, exonic sites (i.e., directly selected sites) were masked, and the summary statistic calculations as well as inference is based only on neutral regions (see Methods). As shown, demographic and selection models can be fit to all datasets, often resulting in strong mis-inference when the assumptions underlying the estimation procedure are violated. 
True

scenario

Summaries of the data

SFS

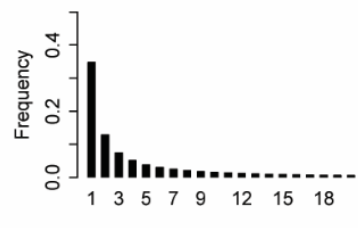

+ BGS

+ Pos

993

994

995

996

997

998

999

1000

1001

1002

1003

1004

1005

1006

1007

1008

1009

1010

1011

1012

1013

1014

1015

1016

1017

1018

1019

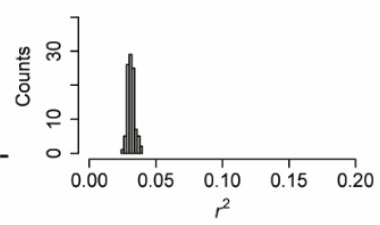

LD

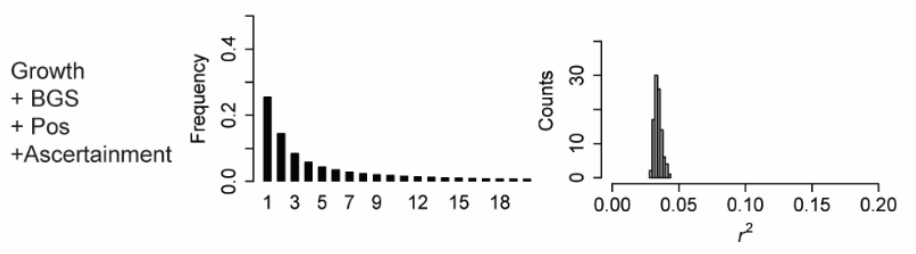
inference.
Fit of simulated data to:
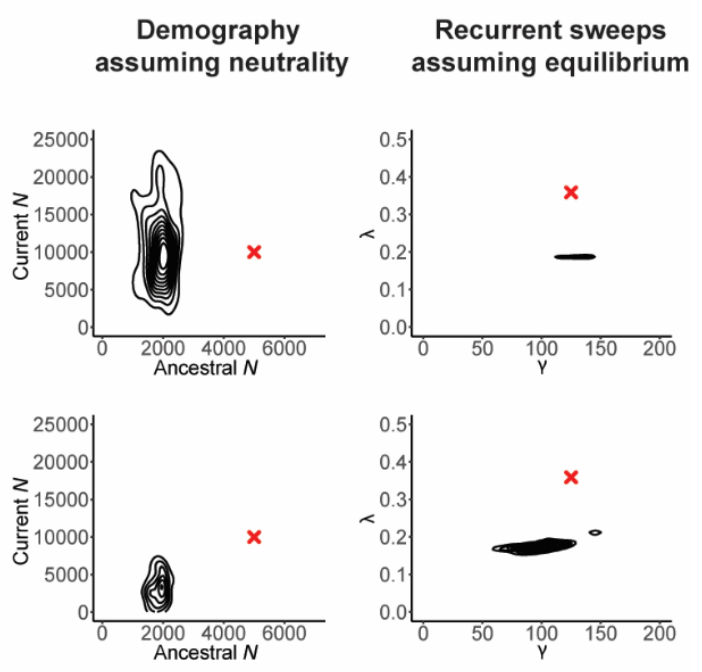

Figure 2. Ascertainment errors may amplify mis-inference, if not corrected

As in Figure 1, the scenarios are given in the first column, here population growth with background selection and recurrent selective sweeps ('Growth + BGS + Pos'), as well as the same scenario in which the imperfections of the variant-calling processes are taken into account - in this case, one-third of singletons are not called ('Growth + BGS + Pos + Ascertainment'). The middle columns present the resulting SFS and LD distributions, and the final panels provide the joint posterior distributions when the data are fit to two incorrect models: a demographic model that assumes strict neutrality, and a recurrent selective sweep model that assumes a constant population size. All exonic (i.e., directly selected) sites were masked prior to analysis. Red crosses indicate the true values. As shown, unaccounted for ascertainment may contribute to mis- 
True scenario

Eqm

$+\psi=0.05$

Eqm

$+\psi=0.1$
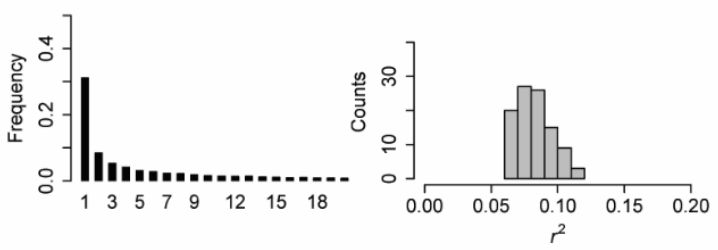

Summaries of the data

LD
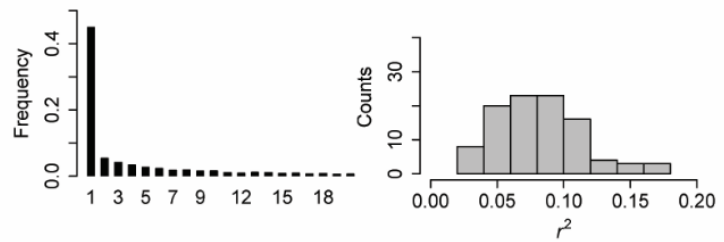

Fit of simulated data to:
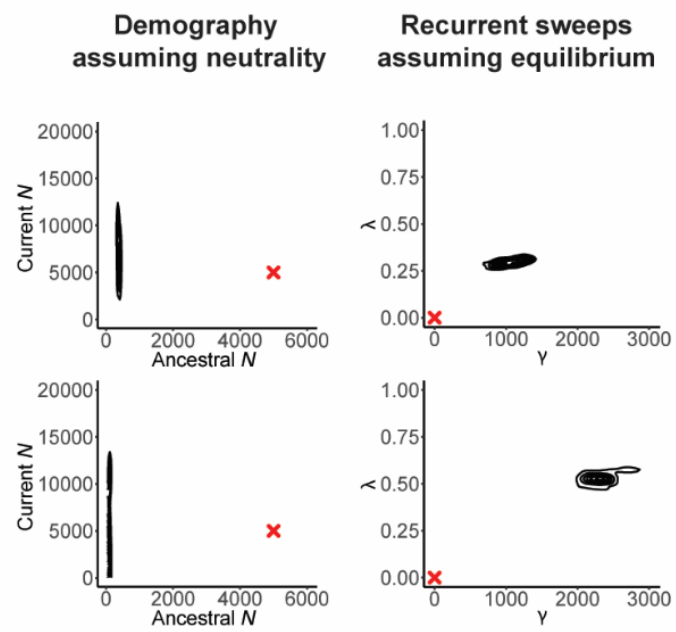

Figure 3. The impact of potential model violations can be quantified

As in Figures 1 and 2, the scenarios are given in the first column, here equilibrium population size together with a moderate degree of progeny skew ('Eqm $+\psi=0.05$ ') as well as with a high degree of progeny skew ('Eqm $+\psi=0.1$ ') (see Methods); the middle columns present the resulting SFS and LD distributions, and the final panels provide the joint posterior distributions when the data are fit to two incorrect models: a demographic model assuming neutrality, and a recurrent selective sweep model assuming equilibrium population size. Red crosses indicate the true values. As shown, this violation of Kingman coalescent assumptions can lead to drastic misinference, but the biases resulting from such potential model violations can readily be described. 
True scenario

Demography assuming neutrality

Fit of simulated data to:

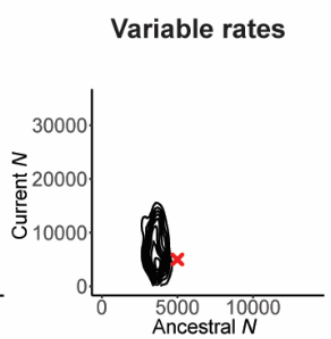

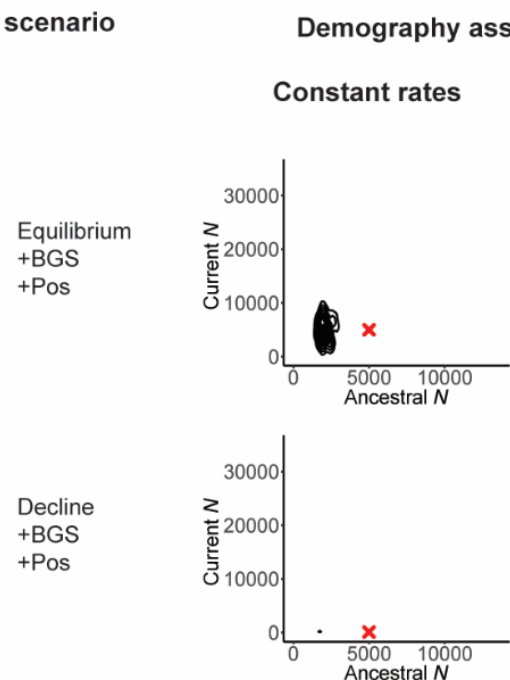

Decline

+BGS

+ Pos

Growth
+BGS

+ Pos
1041

1042

1043

1044

1045

1046

1047

1048

1049

1050

1051

1052

1053

1054

1055
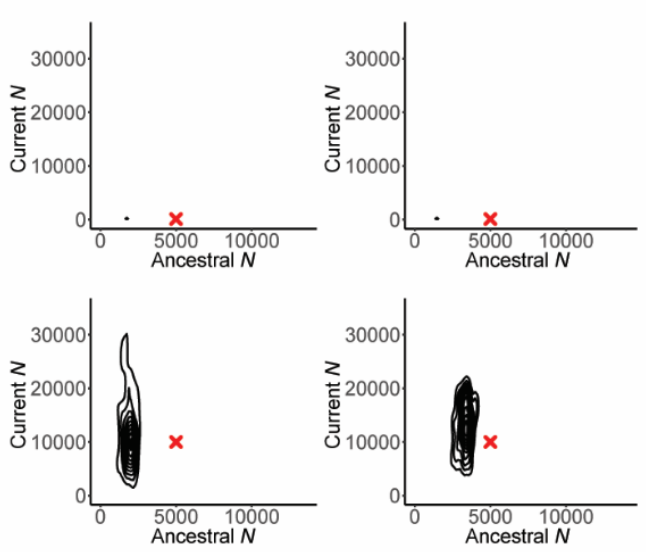

Recurrent sweeps assuming equilibrium

Constant rates

Variable rates
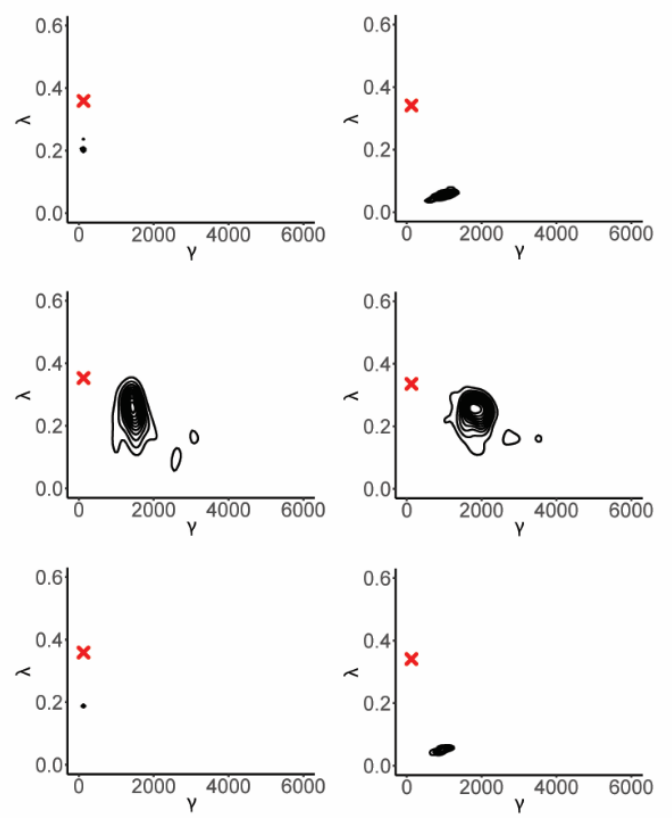

Figure 4. The effects of not correcting for mutation and recombination rate heterogeneity Three scenarios are here considered, equilibrium population size with background selection and recurrent selective sweeps ('Eqm +BGS + Pos'), declining population size together with background selection and recurrent selective sweeps ('Decline + BGS + Pos'), and growing population size together with background selection and recurrent selective sweeps ('Growth + BGS + Pos'). Inference is again made under an incorrect demographic model assuming neutrality, as well as an incorrect recurrent selective sweep model assuming equilibrium population size. However, within each category, inference is performed under two settings: mutation and recombination rates are constant and known, and mutation and recombination rates are variable across the region but assumed to be constant (see Methods). Red crosses indicate the true values, and all exonic (i.e., directly selected) sites were masked prior to analysis. As shown, neglecting mutation and recombination rate heterogeneity across the genomic region in question can have an important impact on inference, particularly with regards to selection models. 